\title{
USO DE LA ESPIROLACTONA EN EL TRATAMIENTO DE LA INSUFICIENCIA CARDIACA CONGESTIVA
}

\author{
Dr. ALBERTO DRAYER B. y Dr. HFRNAN VLLMCIS (4) \\ Deputinruento de Curdiologí, \\ Hospratut Universitarta, Caracus
}

Ell advenimiento de les churóticos derivados de las lyenzotialdiazinas y el mejor conocinriento de los trastornos electrulitions en la insufiriencia caldlaces congestive venusa r-:-7-16 ha sigwijicado un prosteso imporlante en w verlamicnto de estos pacientes. Sin crubargo hay alguros enfelmiss on Ios guc a pesír de los esfuer»us terapúc licos to se ohtime la compensnción

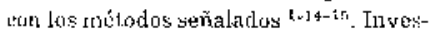
l:igrddses han encontrado que entre lis susturu:as responsables del metabotismo del agsa $y$ del soldio, la aldoslerona ocupa un piesto impostante. fin diverses estadess patolúgions, cumo instuficiencia cardhux'a congestiva, ne-

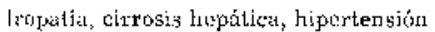
itclesia!, etc., la sidi aislada e identific'ada en la simgre $y$ or:nil, cantidades

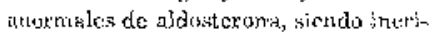
miracla está hormona como la respont::atrle, en gras partes, de la presebriá ab las estadtes edematusos, "-11-1.. Este liecho ha couducido a la búsiquedit y

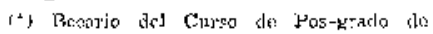

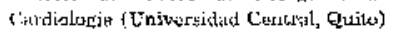

entayen de sumitancias blocineadoras de ]: aldosterinta. 2nieso. Destere 1955 diversiss investigrarlores hin trabajado ( roluctonas, hisia que en 1957, Cella ", Kagawa "y Liddle ", ensalydun 12na spiroílitona capas: de bluqucar lus:

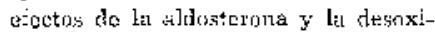

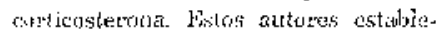

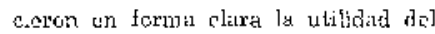
prorlucto, ell el trataniento de estados elemitusius, refraciarios a las medidas

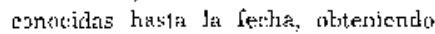

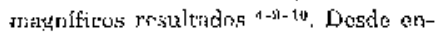
tonees son numerosos los treabajos pun blicadis en relación al tratidrjento cona antiald drsteronas en viurius estadlos ellemalusos 3-r. jucluyerda la insulicientia cardiked refracter a li mal.uralezíz de este trabajo no insistireit)us en el análisis da estas publjcracionen, ture se encitontran al alcunce fâm cil de śos inleresiatdıs, Ds convemienk: stñular, como ha sido curriunicado por Vogt, los siguiestes principius anue debers ser texidos en cuentu on la terizin con lil sustancia que ensayaunos (Nlductoiv) a) qu: ésta produce diuresis $y^{\prime}$ 


\begin{tabular}{|c|c|c|c|c|c|c|}
\hline Cis5o No & 1 & 2 & 3 & 4 & 5 & f \\
\hline $\begin{array}{l}\text { Gis'udte de } \\
\text { Intuficione'tu }\end{array}$ & ill & $1 \mathrm{~V}$ & X]1 & IV & JII & III \\
\hline Diegunstico & $\begin{array}{c}\text { AI. Ao. } \\
\text { Tri. } \\
\text { Reizเnat. }\end{array}$ & $\begin{array}{c}\text { M..Ao. } \\
\text { Tri. }\end{array}$ & $\begin{array}{l}\text { Mlive. } \\
\text { Crev1. } \\
\text { Ch. }\end{array}$ & $\begin{array}{l}\text { Mise. } \\
\text { ciron, } \\
\text { Cly, }\end{array}$ & $\begin{array}{l}\text { I. As. } \\
\text { [. }\end{array}$ & (i.) N.C. \\
\hline $\begin{array}{l}\text { Edtud } \\
\text { años }\end{array}$ & 39 & 32 & 47 & 64 & 54 & 40 \\
\hline Sexo & M & $F$ & $\mathbf{F}^{\prime}$ & liI & MI & IM \\
\hline $\begin{array}{l}\text { Totu] } \\
\text { Días } \\
\text { corstrill }\end{array}$ & 33 & 42 & 33 & 50 & 34 & 22 \\
\hline 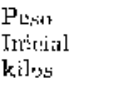 & 47,5 & 59,7 & 47 & 69 & 55,3 & 88 \\
\hline $\begin{array}{l}\text { Pusso } \\
\text { fintal } \\
\text { kiloss }\end{array}$ & 47 & 39,7 & 40 & 60 & 53 & 75 \\
\hline $\begin{array}{l}\text { P.V. } \\
\text { Inicial }\end{array}$ & $\begin{array}{r}230 j \\
350\end{array}$ & $\begin{array}{c}540 ! \\
7041\end{array}$ & $\begin{array}{r}330 ; \\
\quad 420\end{array}$ & $\begin{array}{c}250 / \\
310\end{array}$ & $180 / 290$ & $180 / 260$ \\
\hline $\begin{array}{l}\text { P. V. } \\
\text { f:nal }\end{array}$ & $\begin{array}{r}150 / \\
210\end{array}$ & $\begin{array}{r}150 / \\
200\end{array}$ & $110 \%$ & $\begin{array}{l}120 ! \\
180\end{array}$ & $\begin{array}{l}150 \% \\
250\end{array}$ & $\begin{array}{l}100 / \\
150\end{array}$ \\
\hline
\end{tabular}

natriuresis solamentc cuando existe. hiperaidosteronismo y b) no suprine la produccion de aldosterona pero contrarresta los eflectus a nivel local 24.

MATERIALES Y MLTODOS

Je acuerdo con la Dirseción del
Hospi\{al Uiniversitario de Caracas, se clecidió hateer un ensátyo clínico con spirciactora (Aldattone G. 1), Searle \&: Cu.) para ver la importancia y las upliciciones ric este mediramento en rliestiru nedio hospitalariu.

Sc ascogieron pacientes en insufjcisucia cardjaca congesliva venosá de 


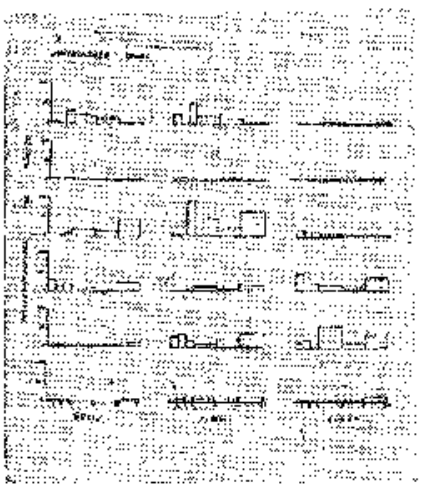

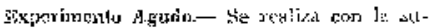

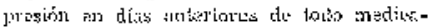

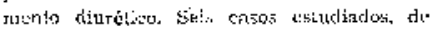

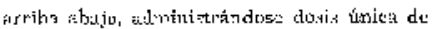
Gentrig. Le Aldactone, a las 6 a: m, tee recogun

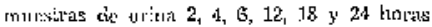

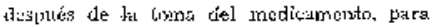
ciosilicaciór de cetstolitos Sudjo. Potesio y Cluri, valoredos en miljequixal:utes miruto par"i sitila mures!nta.

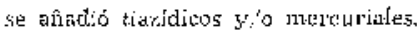

bin tsilis prisferes lases, se ukilizó A]dactone sirli, como únici, nedicemento con indicación distétical, ix stíis the las condiciones bétsicas de ropnso, dieta $y$ djarial, suprimiendis los otros dgentes diuréticus artcriornente sm. pleados y que se hibíin mustrado elíת:ramente incficices, para observar it al Aldactunia e:a capaz pox sí soli' de

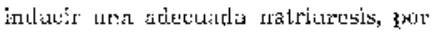
เ:Jumnil de! nivi? de ingestal diariet de udio. Qurríamos nbservar si un vez

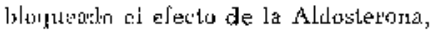

que presultílidus elevacla lj-i-1i-1:; al agregat diurditicos merturiales ys t:azídiroc, sc: incu'e:nentalua o nó, có los zidvoics ob:ejidos com el Alácton? la cairesum de agut y sodio, llevantó a li mejoría de los pacientus. Tla sit muy inforlante este frocedimien: pron tenostrar la selectividat de indicación de la spirulactona.

I'n risestro cstudio, al comenzis administruteión de Aldactone, dentí de lïs pimorus dias, hubo un aumen te la excre:ción de sodio, por encirnt

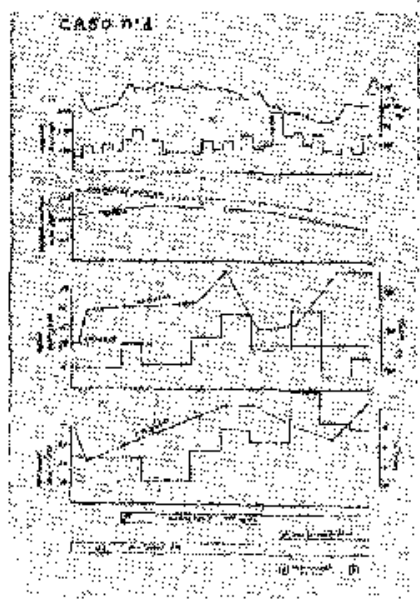

C.sso No 1. Cordiopatia reumálical, mit.

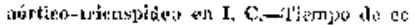

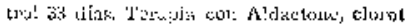

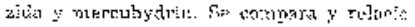

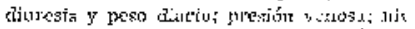

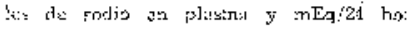

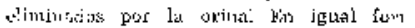
dusiftumirnes de pobiaso.

\section{Biblioteca Nacional del Ecuador "Eugenio Espejo"}




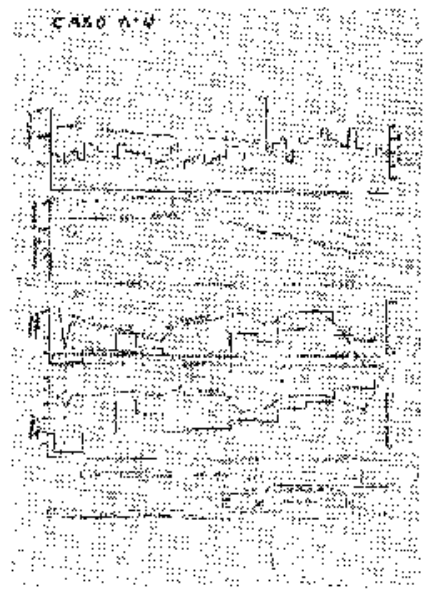

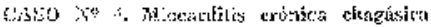

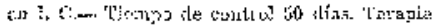

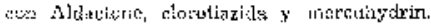
Se compale, y trectacona diuresis y paso diz-

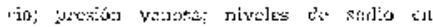

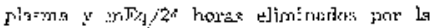

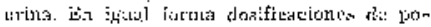
istis?.

eiminación de! sadio y ugua dumentó

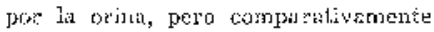
ex xjotes menorus qute los atros casts. Hil resuliarin vis'o en esie paciente: a pesiti del prolonsado sersitro de sidmiulistración del Altactone (34 días), tué menos exilus raje en this casiva combiנ:arisas.

En ol tasu No 6 sn asució Altactone

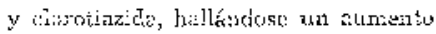
s.gnificido de lia excroción de: sulit, yeru tareblión menos iallyoriente gue ел Ios antariores. Drbomos anotar quo

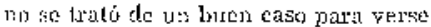
eforis ditaríticus, ya que se trataba do wua olowerữonel'ritis crónica, con maijientacinnes dr: insulictenceia rewal Lrónteda.

\section{DISCUSION}

['é. ostul:o ane homes electuado,

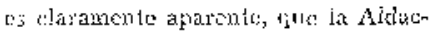

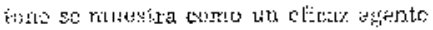

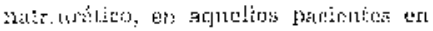
quie los dilláticos mercinia ís y tiat-

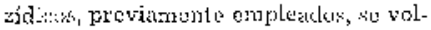

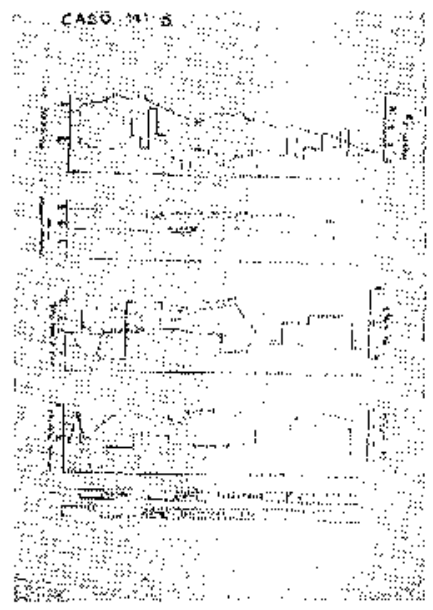

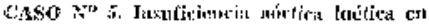

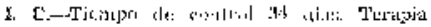

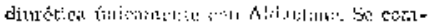
para $;$ 小!

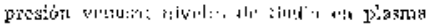

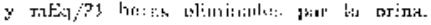

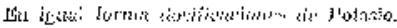


vimon inelicacos, siendo estr una domuxliación indirecta de la existencia rle tenores elevados de aldosterona en of uxganismo, i-1f-it-15. Es sabidio por invostigaciones realizadas por varios datores, que en tasios donde hay ederna y ruy partícularmento cn $I_{\text {át }}$ insuficíencia cardísca, la aldosterone puede enenutrarse en enncentraciones nocivamente altas para la climinación del rodio por los tribulos renales, debido a una falla del mecanismo de la des-

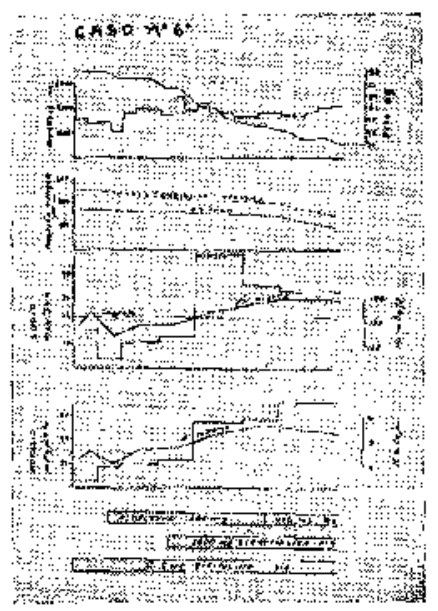

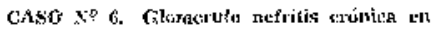
I. C.F inzidir:iencia revial crón:ea. Tirmpo de contiol z2 dias. Torapis cliurólica car A.'dactom $\mathrm{y}$ clorotiaz:cij. Se compora $y$ rela-

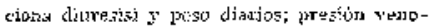
s.; givelne di Sodio s. foras climinados por lie oritia. En igual fornu dosifjercjones de Potasiu. trucción de esta sustancia 1.6 o por uma cxagerada estimulación eu su prow ducción 1는.

Igualmente podemos ver, de Ios exporingntos y ubservaciones realizadas, que no judemos considerar al Aldaclone entno un potenle slatriluretice, ya que cuando fue driministrado sólo, coma únicu meticamento, los alumentrs cell la txeroción de isdio, nis son en nudo alguno, cumparables con los que s0 olotienen en pacientes con bueja res. puesta a lus diuréticus marcurules $y / 0$ tiazílicos.

Es importantc el señalamiento de

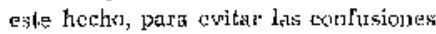
y equímcas en cuasto a la especif:eitad leraprutica del Aldantone, siesdo indisporsable deslacar, que a los pacien os, a los que se administró este medicameato, no habisin lespoudiclo a la terapia corrientemente cripleada, por lo que tis duduce, que su jndicación absolute $y$ precies es euando no se estin obtenjendo buenos resultados cun el empleo de los disurétios de liso habitual,

Int nulasiros experitrseltos agtudos obsurvarnys, on etatro de los tesos exiludiadirs. que hacia las is y 24 horis después de administrarlo el medicamenlu, se prorlusía un sumento da ly

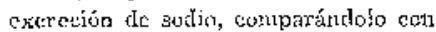
las canticlades de Ius horits intermedias. Pura aste frórruse prodemus aportar dus hipotesis: a) una prolongación del efecto lerapéutico de la spirolactona, más aljá de lias 18 horus de a administrución; $y$ b) la pasibjilidad cie que al bloquearsc parte del exuraj de la aldoslerona, quedej en libertat 
de manifestarse los itecanismos satri-

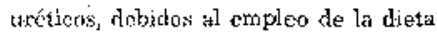
restrictiva de sudio, del reposo $y$ dig:thl, frenados o inhibidos por el aldostcrorismo scrundario existente.

Otro hechu digno de destacarge, es la observición de la peesistencia de in natriumesis y to ba vuetta a la eficiacia de los dincíticas mercuriales y/o tiazídicos on los paciontes, después de da administración durante algún tienupo do la Aldactore, unnque cista sea supriruida, pudićndose internzetar este hecho, corno la ruptiva de un círculo vicisso que existín en el onfermo, por mecanismos de hiperaldosterobismo secundario. 12-15.

Na queda duda de la gran utilidarl de las spirolactonas en el trataniento de los estadus edematosos y muy partixularmente en in insuficiencia eardíaca congest:va, sicmpro y euardo su emplen se harga con la precisión que esite medicimento reclamis.

Esta ha sido la razón de haber efectuado este trabijo y por lo que se ha dikfo primenrismenle Aldactone como única terapia diurćlice, aserizindole posteriormente a los otros augentes jiurécicos an reterencia, para que quede demostride, que ćlla es capaz de inducir diuresis euandr nu se olstenía, pero su putencid cotwo agento uatriuretico per se es más bien medivcre, pir lo cuat nu se justifíla su cappleo sólu, ni mucho jnenos substituix a los otros agentes ratriuréticos por éltá.

No entramos en considerariones de orien farmacológico por escaparse a Ios Jímiles de este tubajo y por considerar que élias han sido ampliamente expuestas en trabajos especializadi sobre la matería ${ }^{1-0-1 k=1 !}$.

\section{PHSUMIEN}

En el presente trabajo se ensayó b spirolactona (Aldactona) un antagoni. ta de la aldosterona, en el tratamien: de varios pacientes con insufieienc. cardíacx con'sestiva.

Se sulecionaron veis pacientes co 1V. en quienes la terapin habitalal i: imsuficiencia congestiva, grados III: habia redurido completamente el edt. ma. La administración de uns atosi únicit de Aldactore (600 mg.) zyeodut: y nuáx tardía de la eliminación de sodu! aumento de da diuresis por 12 hor't

In los mismos pacientes, la adminis: tración costidiana de 3 a 4 dosis de 201 mis. produjo aumento inicia] de la el. minación de sudio, la misma que posit: fiormejto aumentí snás al agregar o ¿ratamiento un durćtico.

\section{SUMLARY}

Spirolactone (Aldtetone) was asta $y$ ed in the treatsment of patienl.s witl: corrgestive heart"faiture fgrade III ani IV).

Six pationts vere selected becaua the usual treatment wish eardiotorit agrents and diurretics ferled to ro. duce the edemas. The administration of a single $600 \mathrm{mg}$ dose of spiralactone produced a inatked incrasse of the dits. resis during 12 hours, Inctesse in the chimination of sodium appeared latex.

In the same putionts daily adminis 
Irulicun of 3 to 4 doses of $200 \mathrm{mg}$ each, also resulted in increasings the elimiuntion of sodium which was potentiaInd by the addition of a thineidic diuriti:c.

\section{BIRLLOGIRAFIA}

I.-BFYFR, K. II., Jr.: Recent advaruecs in the knowledge of the eauses of edema and in clituctic therpay: non moteurial orpanic diuretica,A. MI. A. A,ril. Int. Mrcd. 102:1005, 1958.

¿-BLANCO, P., Drayer; A., Henriquez, S.: Estudio comparativo de la clorotiezida con otron agentas diuréticios, Arets. fest. Cardiul. Nlex. 29: [72, ]1950.

:I-_DOLi'E, E. and col: Siuties on new diuretic compounds: spirclactons and chloratiazide, Cand. M. A. J. 79:881, 1958.

A. CEELLA, J. A. and Kayawa, C. MI.: Šbu roidal lartores, J, Ar. Chem. Spe. 79:4808, 1957

5.-CONN, J. W.: Evolucion of prlmary aldosterontism as a highly specifie entity, J. A. M. A. 172:1850, 1980.

B-CONN, J. W., and other: Metabolic effeets is musenat mes and in primary aldosieronistn of a synthetic "Aldostewne Antagonist", J. Lab. \& Clin. Med. 52:805, 19158,
7.-DRAYER, A., Blanco, R., y Henrifuey, Là cilurotiazida somo agentes rirrétlco en

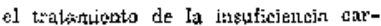

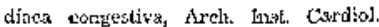
Me: $x, 29: 533,1959$,

8.-EDMOKDS, C. J.: An aldosicrone antasparist and cliuretics in the treatinent of vruitie edema and ascitis, Lancet 1:509, $3 \$ 00$.

9.- KAGAWA, C. M., Cells, J. A., and V:tr A:man, C: G.: Action of new slerold In ilocking effects of aldosterone and desnxycorticozterone on salt, Scionee 126:1015, 1957.

10.-KAGAWA, C. M., Stublevant, F. M., and Yatt Arman, C. G.: Ptsercmatology of ti J:ew steroild t!lat blocks salt aclivity of aldosterone atsel desoxycarticosisent. J. Pharmacol. \& Expcr. 126: 127, 1939.

11.-TLDDLE, G. W: Sodjus diurwsis inchuwed by steroidal antagonist of aldasterone, Science 126:1016, 1957.

12.-TALSO, H. J.: Antzgonistas ic la aldosterone, Clin. Mod. Nort. Awss. pás. 33, Enc. 19G3, Ed. Interamerítara BIex.

I3,-TLIRLIN, I. N. and others: Trivglinent of edema with orally adminisbred spirolictone, J. A. M. A. 174:869. 1960.

14.-Votri, A.: Four Jerads of diuretic therapy, Ant. J. Cordial. 8:11178, 196\%.

15.-WOLFT', H. R., Kocsorek, K. R., and Guehlsnrh,; HLyporalinostersoism in heart clisease, Laredt 2:63, 1957. 